\title{
Enrutamiento y asignación de longitud de onda: una solución con búsqueda Tabú en tráfico dinámico
}

\author{
Routing wavelength assignement: a solution with Tabu search in dynamic traffic
}

\author{
Arturo Rodríguez García $\quad$ Leonardo Ramírez López ${ }^{2} \quad$ Luis Rivera Méndez ${ }^{1}$ \\ Antonio Gutiérrez Osorio ${ }^{1}$
}

Recibido 4 de diciembre de 2013, aceptado 5 de mayo de 2014

Received: December 4, 2013 Accepted: May 5, 2014

\begin{abstract}
RESUMEN
El aumento de la demanda de servicios por internet ha generado la necesidad de aumentar la oferta de ancho de banda y el acceso oportuno a los servicios de transporte en las redes ópticas con tecnología WDM (Wavelength Division Multiplexing). Sin embargo, los algoritmos heurísticos como algoritmos genéticos, Simulated Annealing, asociados a brindar este servicio, no logran un desempeño óptimo bajo alta carga de demanda en condiciones de tráfico dinámico. El presente trabajo propone una estrategia integral para solucionar el DLE (Dynamic Ligthpath Establishment), es decir, sin dividir el problema, utilizando el algoritmo Búsqueda Tabú. Los resultados muestran que el algoritmo utilizado responde mejor en escenarios dinámicos, pero con una alta utilización de la red.
\end{abstract}

Palabras clave: Recocido simulado, algoritmo genético, longitud de onda, búsqueda Tabú.

\begin{abstract}
The increase on the demand of online services has generated the need to increase the supply of bandwidth and timely access to transport services in optical networks with WDM (Wavelength Division Multiplexing) technology. The algorithms associated to provide this service, do not achieve an optimal performance under high demand load in dynamic traffic condition. The present paper proposes an integrated strategy for the solution of the DLE (Dynamic Ligthpath Establishment), that is, without dividing the problem, using the Tabu Search algorithm. The results show that the algorithm used works better on dynamic sceneries, but with a higher network utilization.
\end{abstract}

Keywords: Simulated annealing, genetic algorithm, wavelength, Tabu search.

\section{INTRODUCCIÓN}

El aumento de la demanda de servicios y el desarrollo de las aplicaciones de internet tales como IPTV, Cloud Storage, y Peer to Peer (P2P) entre otras, requieren una gran cantidad de ancho de banda y soporte comunicacional. Sin duda actualmente las plataformas encargadas de transportar dicha carga son las redes de fibra óptica con WDM (Wavelength Division Multiplexing) y su evolución hacia DWDM (Dense Wavelength Division Multiplexing) [1], explotada con el protocolo MPLS (Multiple Protocol Label Switching). En este ámbito, la discusión se centra en la conmutación (Switching), la que es preferible debido a su alta velocidad de respuesta frente al enrutamiento (Routing) [2]. El incremento

\footnotetext{
1 Departamento de Tecnologías Industriales. Facultad Tecnológica. Universidad de Santiago de Chile. Avenida Ecuador 3659. Estación Central. Santiago, Chile. E-mail: arturo.rodriguez@usach.cl; luis.rivera@usach.cl; antonio.gutierrez@usach.cl

2 Facultad de Ingeniería. Universidad Militar de Nueva Granada. Bogotá, Colombia.

E-mail: leonardo.ramirez@unimilitar.edu.co
} 
de la capacidad de transmisión de las redes WDM para las comunicaciones del futuro genera que las capas superiores de OSI se acerquen a la capa enlace. Lo que lleva a la reflexión sobre la permanencia de la capa IP asociado al enrutamiento. Sin embargo, lo más probable es que la dirección IP permanecerá como identificador de puntos o nodos de red. En cualquier caso, la búsqueda actual es mejorar el establecimiento de rutas que determinen una conexión rápida y segura. Se deberá resolver con los diferentes algoritmos ya existentes [3], sin dejar de innovar en nuevas heurísticas que apoyen en este ámbito para resolver los problemas que la demanda futura trae consigo. Por otro lado, la demanda óptica requiere de buenas rutas más que de rutas óptimas; esto se debe a la necesidad de disminuir la probabilidad de bloqueo de la red y mejorar la utilización de la red.

El tráfico que soporta una red puede ser clasificado como estático y dinámico [4-5]. En las redes ópticas de transporte el tráfico tiene un problema denominado RWA (Routing Wavelength Assignement), en la actualidad la relevancia de este problema se ve incrementado debido entre otras cosas al aumento de la demanda de servicios.

Se requiere seleccionar una ruta y una longitud de onda para establecer la conexión, bajo la restricción de que no exista cambio de longitud de onda a lo largo de la ruta seleccionada, esto se conoce como CCW (Continuity Constraint Wavelength) [4]. Esta restricción ayuda a mejorar el retardo que ocurre cuando se utilizan diferentes longitudes de onda en la misma ruta [5]. Pero se genera un aumento en la utilización de la red aumentando la probabilidad de bloqueo de la demanda futura, ya que los caminos se agotan mientras más rutas se establezcan. Se han desarrollado sistemas de enrutamiento con reutilización de la longitud de onda, donde se permite cambiar la longitud de onda en los enlaces, sin embargo los estudios no muestran cambios significativos en el rendimiento del enrutamiento [11]. Por lo general, este problema se ha resuelto subdividiendo el problema en dos partes, la primera parte resuelve la ruta a seguir basado en minimizar alguna condición preestablecida; la segunda parte resuelve el problema de asignación de longitud de onda por medio de algoritmos como First Fit (FF), Next Fit (NF), More Fit (MF) y Random Fit (RF), entre otros [4-6]. Existen otras propuestas que resuelven el problema desde una perspectiva integral (sin subdividir) [7-9]. El presente trabajo realiza el estudio del desempeño de Tabú Search comparándola con otras heurísticas, utilizando como indicadores la probabilidad de bloqueo y la utilización de la red, simulados en la red National Science Foundation NETwork (NSFNET).

\section{DESCRIPCIÓN DEL PROBLEMA RWA}

Las tecnología WDM se ha desarrollado rápidamente en las redes ópticas multiplicando el ancho de banda para dar servicio a las capas SDH/SONET (Synchronous Digital Hierarchy/ Synchronous Optical NETwork). La red óptica es demandada por solicitudes de conexión que deben traducirse en rutas y longitudes de onda asociadas. Las redes ópticas están formadas por conmutadores denominados OXC (Optical Cross Connect) y enlaces de fibra óptica entre ellos. Las rutas están formadas por uno o más enlaces entre $\mathrm{OXC}$ de la red. La información transita por los enlaces en señales lumínicas de distintas longitudes de onda, es decir, a diferentes frecuencias. En la Figura 1 se muestra una parte de la red óptica, donde los OXC se han subindicado con $\mathrm{x}, \mathrm{x}+1, \mathrm{x}+2, \mathrm{x}-1, \mathrm{x}-2$ para diferenciarlos, y las líneas continuas indican las señales lumínicas de diferentes longitudes de onda (Lambdas). Dependiendo de la tecnología de los OXC, estos podrán conmutar fibras, longitudes de onda, bandas de longitudes de onda, canales de tiempo TDM (Time Division Multiplexing), para esta investigación se conmutarán longitudes de onda. Las rutas ópticas están formadas por enlaces, los que direccionan el haz de luz (datos) mediante la misma longitud de onda según la restricción de

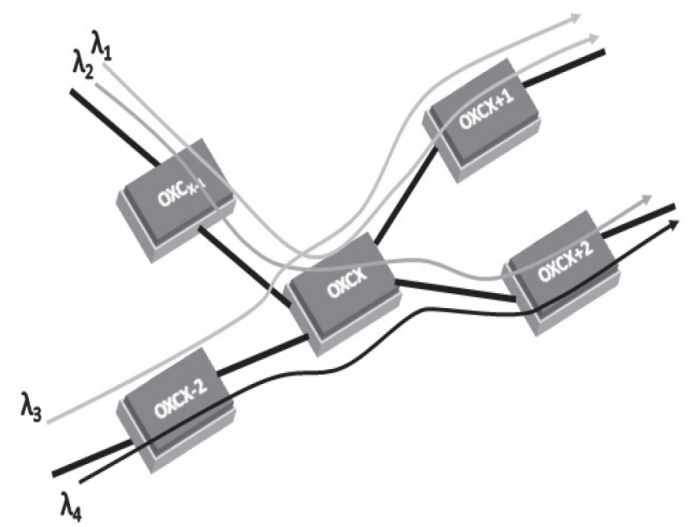

Figura 1. Ejemplo de conexión en redes ópticas. 
continuidad de longitud de onda (CCW), la dupla formada por la ruta $\mathrm{R}$ (conjunto de enlaces) y la longitud de onda $(\lambda)$ determinan el par $(\mathrm{R}, \lambda)$ que es denominado LigthPath (LP) [10].

Sin embargo, la posibilidad de encontrar un LP disponible se agota cuando la demanda supera a la disponibilidad y es cuando se bloquea la solicitud.

En la Figura 1 se observan cinco OXC, que forman parte de un sistema mayor; las rutas establecidas (LP) con su longitud de onda respectiva son: $\left(\mathrm{x}-1, \mathrm{x}, \mathrm{x}+2, \lambda_{2}\right) ;\left(\mathrm{x}-1, \mathrm{x}, \mathrm{x}+1, \lambda_{1}\right)$ y $\left(\mathrm{x}-2, \mathrm{x}, \mathrm{x}+1, \lambda_{3}\right) ; \mathrm{al}$ llegar una solicitud de servicio cuya solución pasa por los enlaces $(\mathrm{x}-2, \mathrm{x}, \mathrm{x}+2)$ no se le podrá asignar la longitud de onda $\lambda_{1}$, debido a que se encuentra en uso en el enlace $(\mathrm{x}, \mathrm{x}+2)$, por lo que se deberá utilizar otra longitud de onda o, en su defecto, buscar una nueva ruta. Esto se denomina contención.

\section{DESCRIPCIÓN DEL MODELO DE DEMANDA}

En la red existe un tráfico actual, y una demanda futura o solicitada; el problema RWA se denomina SLE (Static Ligthpath Establishment) si el tráfico es estático, y DLE (Dynamic Ligthpath Establishment) si el tráfico es dinámico [5]. El tráfico es dinámico cuando el tiempo de conexión promedio solicitado $\left(t_{C}\right)$ es menor que el tiempo promedio entre llegadas $\left(t_{L L}\right)$ de la solicitud de servicio, como se observa en la Figura 2.

El escenario de tráfico dinámico no es optimizable (NP-Completo), por lo que se utilizan algoritmos heurísticos; que en su mayoría ofrecen mayor cantidad de soluciones no necesariamente óptimas.

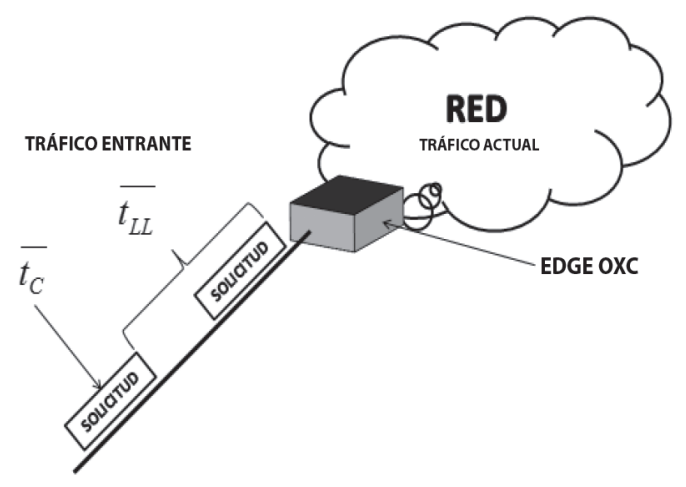

Figura 2. Variables de tráfico.
La complejidad computacional es un elemento importante en este problema, y al subdividir el problema se logra disminuirla, debido a esto último está presente en muchas investigaciones. Por otro lado, las múltiples rutas que obtienen los algoritmos heurísticos resultan de gran apoyo cuando se presenta el problema de contención, evitando de esta forma una nueva ejecución del proceso algorítmico. Las características de las variables de demanda son similares a los utilizados en los trabajos [3-5].

\section{Demanda de la red}

A los nodos fronterizos (Edge OXC) llegan solicitudes con distribución Poisson, sin embargo se podrían estudiar diferentes escenarios probabilísticos. Dichas solicitudes traen consigo 3 parámetros que deben ser satisfechos, de lo contrario la solicitud deberá ser bloqueada.

$$
d_{i}^{S}=\left(r_{O}, r_{D}, n_{C}, t_{C}\right)
$$

En la ecuación (1) se definen:

$d_{i}^{S}$ Es el vector que representa la i-ésima demanda que llega al s-ésimo nodo fronterizo.

$r_{O} \quad$ Es el número de identificación del nodo origen de la demanda entrante.

$r_{D} \quad$ Es el número de identificación del nodo destino de la demanda entrante.

$n_{C}$ Número de conexiones solicitadas hacia $r_{D}$.

$t_{C}$ Tiempo de conexiones solicitada para el par $\left(r_{O}, r_{D}\right)$.

El problema es establecer las rutas y la longitud de onda, observando los indicadores utilizados en los trabajos [3-5], como mínima probabilidad de bloqueo de solicitudes y utilización de la red.

\section{DESCRIPCIÓN DE LOS ALGORITMOS}

El problema es establecer las rutas y la longitud de onda, observando los indicadores utilizados en los trabajos [3-5], como mínima probabilidad de bloqueo de solicitudes y utilización de la red.

El presente trabajo está centrado en el problema DLE, el mismo que ha sido ensayado bajo diferentes estrategias y diferentes heurísticas para satisfacer la demanda de una red óptica, y se han utilizado criterios y algoritmos de optimización que son descritos en $[3,7]$. 


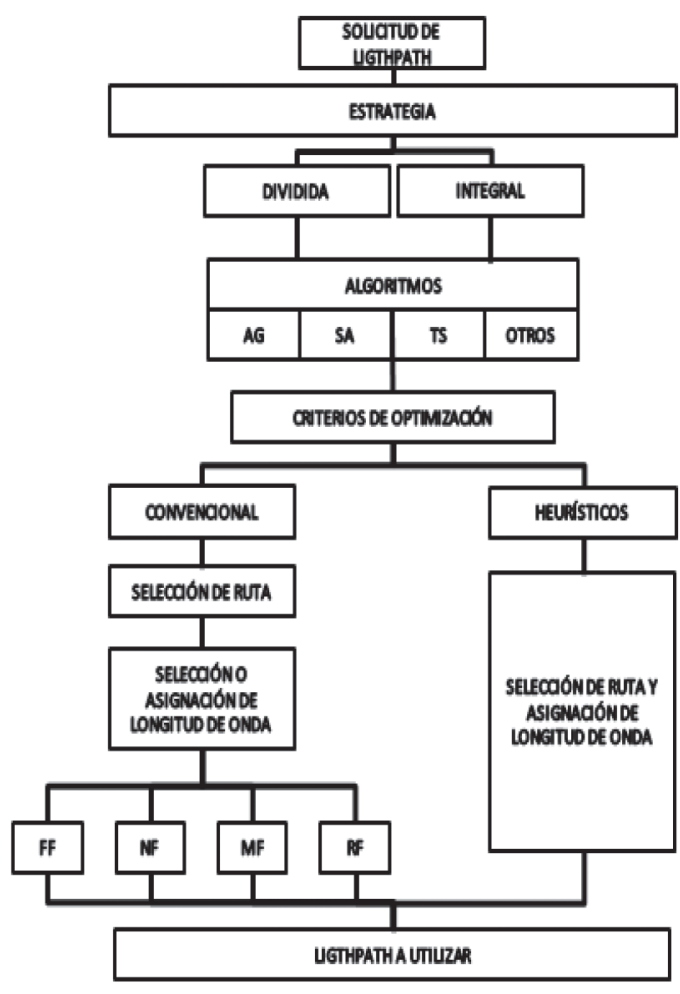

Figura 3. Estrategias de solución RWA-CCW.

La Figura 3 muestra una clasificación de las estrategias encontradas en la literatura para resolver los problemas de SLE y DLE. Los algoritmos heurísticos son muy utilizados en la búsqueda de buenas rutas y no necesariamente rutas óptimas, tales como: Hormiga [12-14], Genéticos [3, 7, 22], Búsqueda Tabú [1, 15-19] y Simulated Annealing [8], todos los trabajos con la estrategia de dividir el problema en dos partes, excepto [3,7-8] que los hace integralmente, es decir, sin dividir el problema solucionándolo en un solo proceso algorítmico.

Como se observa, va desde utilizar estrategias de división del problema hasta resolverlo integralmente, utilizando distintos criterios y algoritmos que permitan obtener una ruta y una longitud de onda, la que permita transportar la información. Cabe resaltar que en redes ópticas no necesariamente se busca optimizar, lo que se busca es una ruta que funcione, sin generar un aumento importante de la probabilidad de bloqueo y sin aumento de la utilización de la red.

Los Algoritmos Genéticos (AG) y el algoritmo Simulated Annealing (SA) son ampliamente descritos en [3, 7-8], procederemos a describir el algoritmo Tabú Search (TS).

\section{Algoritmo Tabú Search o Búsqueda tabú}

El algoritmo TS fue desarrollado por Dr. Fred Glover (1989). Fue hasta 1990 cuando el algoritmo se popularizó para la resolución aproximada de problemas de optimización. La ventaja del algoritmo es que una vez localizada una posible solución converge rápidamente, dicha convergencia se debe a que penaliza aquellas acciones que se alejan de la solución local, conservando siempre vecindades con alta probabilidad de contener una solución. Básicamente se sostiene en la premisa que se aprende más de las acciones erradas, generando información para no realizarlas nuevamente. TS ha establecido nuevos récords en la búsqueda de mejores soluciones a los problemas de planificación de la producción y la programación, asignación de recursos, diseño de redes, enrutamiento, análisis financiero, las telecomunicaciones, la planificación de la cartera, gestión de la cadena de suministro, el modelado basado en agentes, el diseño de procesos de negocio, la previsión, el aprendizaje automático, minería de datos, etc. [20-21].

\section{Modelamiento del algoritmo TS}

Sabiendo que $\mathrm{N}$ es el número de nodos en la red, $n_{C X}$ es el número de conexiones máximo en cada enlace, supuesto uniforme, $n_{W}$ es el número de longitudes de onda en cada enlace, supuesto uniforme. Se han establecido seis matrices, la matriz de enlaces $\mathrm{E}$, la matriz de costos $\mathrm{C}$, la matriz de lambdas $\lambda$, la matriz de tiempos T, la matriz poblacional $\mathrm{P}$ y la matriz de penalización $\mathrm{Q}$, observables en las ecuaciones (2), (3), (4), (5), (6) y (7). La matriz E conservará la topología de la red $\mathrm{E}$, en este caso la red NSFNET. A continuación se muestran las definiciones de las matrices.

Se introdujeron cuatro columnas más en la matriz poblacional con la finalidad de guardar cálculos parciales como costos de rutas y valor de la función aptitud.

$$
E=\left\{\begin{array}{l}
e_{i j} / e_{i j}=0 \forall i=j \wedge e_{i j}=G \wedge i \neq j N E E \\
\wedge e_{i j}=1 \wedge i \neq j S E E \wedge G \gg>n_{C} \\
\wedge i, j \in[0, N-1]
\end{array}\right\}
$$

$S E E=S i ́$ Existe Enlace

$N E E=$ No Existe Enlace 


$$
\begin{aligned}
& C=\left\{\begin{array}{l}
c_{i j k} /\left(c_{i j k}=n_{C X} * e_{i j} \wedge i, j \in[0, N-1]\right. \\
\wedge k \in\left[0, n_{W}-1\right]
\end{array}\right\} \\
& \lambda=\left\{\begin{array}{l}
\lambda_{i j k} /\left(\lambda_{i j k}=0 L A \vee N E E\right) \vee \\
\left(\lambda_{i j k}=k+1 \wedge L D\right) \forall i, j \in[0, N-1] \\
\wedge k \in\left[0, n_{w}-1\right]
\end{array}\right\}
\end{aligned}
$$

$L A=$ Lambda Activada.

$L D=$ Lambda Desactivada.

$$
\begin{aligned}
& T=\left\{\begin{array}{l}
t_{i j k} /\left(t_{i j k}=-t_{c} L A \wedge t_{i j k}=0 N E E\right) \vee \\
\left(i_{i j k}=1 L D\right) \forall i, j \in[0, N-1] \wedge k \in\left[0, n_{w}-1\right]
\end{array}\right\} \\
& P=\left\{\begin{array}{l}
p_{i j k} / p_{i 0 k}=r_{O} \wedge p_{i(N-1) k}=r_{D} \wedge \\
p_{i j k}=r_{X} \quad \forall i \in[0, N-1] \wedge j \in[0, N+3] \\
\wedge k \in[0, N-1]
\end{array}\right\}
\end{aligned}
$$

$$
Q=\left\{q_{i j} / q_{i j}=q \forall i<j \wedge q \geq 3 \wedge i, j \in[0, N-3]\right\}
$$

Donde:

$e_{i j} \quad$ Valor que representa la existencia de enlace entre nodos $i$ y $j$.

$G$ Número muy grande.

$c_{i j k}$ Costo instantáneo asociado al enlace $(i, j)$ para la longitud de onda $\lambda_{k}$.

$n_{C X}$ Número de conexiones por enlace.

$n_{W}$ Número de longitudes de onda por enlace.

$\lambda_{i j k}$ Valor instantáneo que representa la disponibilidad de la longitud de onda asociada al enlace $(i, j)$ para la longitud de onda $\lambda_{k}$.

$t_{i j k}$ Valor instantáneo que representa la disponibilidad temporal de la longitud de onda asociada al enlace $(i, j)$ para la longitud de onda $\lambda_{k}$.

$p_{i j k}$ Valor que representa los nodos en la matriz poblacional que conforman las rutas propuestas para el k-ésimo nodo.

$q \quad$ Valor de penalización superior a 3.

La Figura 4 muestra los procedimientos que se realizan para obtener la LP en un solo proceso algorítmico, lo que garantiza una rápida obtención de la LP, y sobre todo la posibilidad de encontrar más ligthpaths sin repetición del algoritmo.

En las ecuaciones (8) y (9) se define LP, sabiendo que $r_{x y}$, es el enlace existente entre los OXC $x$ e $y$ :

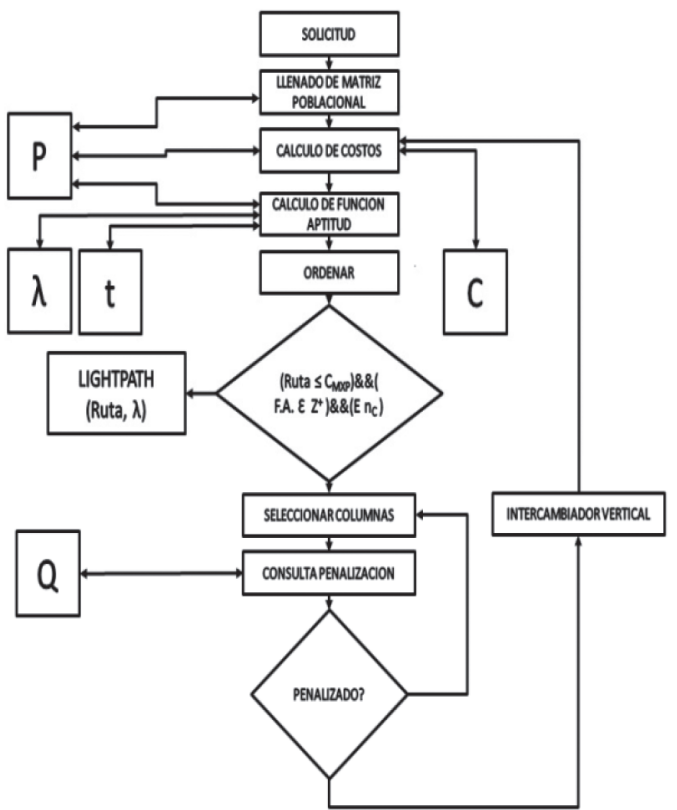

Figura 4. Diagrama de flujo del algoritmo TS.

$$
R=r_{O 1} \bigcup r_{a b} \cup \ldots \ldots . \cup r_{w z} \cup r_{z D}
$$

$$
L P=(R, \lambda)
$$

Por ejemplo, sea la demanda de la séptima solicitud $d_{7}^{1}=(1,4,2,500)$, que llega al nodo etiquetado con 1 , de una red de 8 nodos $(\mathrm{N}=8)$. A continuación se establece la matriz poblacional, donde se resolverá el LP buscado, mayor detalle de la matriz poblacional puede observarse en [3,7-8]. La matriz poblacional se completa de la forma como indica la Figura 4.

A la matriz de la Figura 5 se aplicará el algoritmo TS, y luego se aplicará la actualización en la matriz de costos $\mathrm{C}$ y de lambdas $\chi$, a continuación se procede a resolver el LP a la solicitud del siguiente nodo.

Se procede al llenado de la matriz poblacional con el origen y destino obtenido de las solicitudes, en las columnas 0 y (N-1) de la Matriz Poblacional; reservando la columna $\mathrm{N}$ para guardar los costos instantáneos de las rutas durante las iteraciones, y la N+1 para la función aptitud. Luego se llena aleatoriamente de la columna 1 a la N-2, observar Figura 6.

Se procede al cálculo de los costos de las rutas; por ejemplo, la primera ruta 1-3-1-5-7-5-1-4, los 
$\begin{array}{llllllllllll}0 & 1 & 2 & 3 & 4 & 5 & 6 & 7 & 8 & 9 & 10 & 11\end{array}$

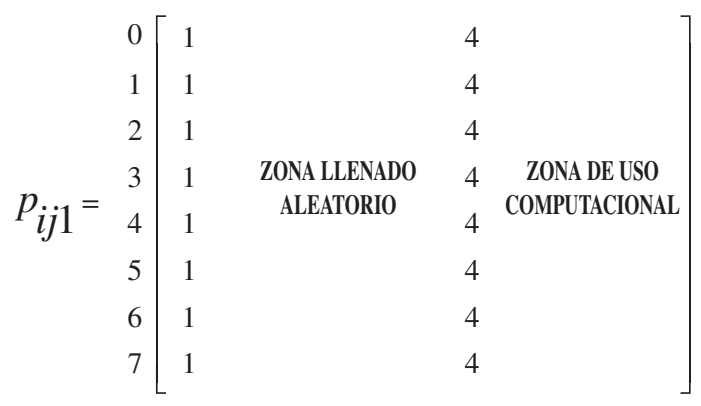

Figura 5. Matriz poblacional para el nodo origen 1, llenado del nodo origen y destino de la solicitud.

$$
\left.p_{i j 1}=\begin{array}{lllllllllllll}
0 & 1 & 2 & 3 & 4 & 5 & 6 & 7 & 8 & 9 & 10 & 11 \\
1 & 0 \\
2 & 3 \\
4 & 5 \\
6 & 7 & 3 & 1 & 5 & 7 & 5 & 1 & 4 & & & \\
1 & 6 & 3 & 3 & 6 & 1 & 4 & 4 & & & \\
1 & 7 & 4 & 6 & 4 & 5 & 5 & 4 & \text { COMPUTACIONAL } \\
1 & 3 & 3 & 7 & 2 & 1 & 3 & 4 & & \\
1 & 2 & 6 & 8 & 5 & 4 & 6 & 4 & & \\
1 & 1 & 1 & 3 & 3 & 7 & 3 & 4 & & \\
1 & 5 & 3 & 6 & 1 & 1 & 5 & 4 & & &
\end{array}\right]
$$

Figura 6. Matriz poblacional para el nodo origen 1 , llenado aleatorio.

costos serán exageradamente grandes debido a que en las rutas algunos enlaces no existirán y tendrán valores grandes. Sea el costo de una ruta $C T$ en el k-ésimo nodo, observar ecuación (10).

$$
C T_{i}=\sum_{j=0}^{N-2} c_{\left(p_{i j k}\right)\left(p_{i(j+1) k}\right)}
$$

Luego, al cálculo de los costos y la función aptitud (FA) y se guardan en las columnas $\mathrm{N}$ y $\mathrm{N}+1$, observar ecuación (11):

$$
F A_{i}=\frac{\sum_{j=0}^{N-2} c_{\left(p_{i j k}\right)\left(p_{i(j+1) k}\right)} \lambda_{\left(p_{i j k}\right)\left(p_{i(j+1) k}\right)} t_{\left(p_{i j k}\right)\left(p_{i(j+1) k}\right)}}{C T_{i}}
$$

Se procede a ordenar de menor a mayor la matriz poblacional, según los costos de las rutas. Luego se procede al intercambio de las columnas de la columna 1 a las $\mathrm{N}-2$; cuando los intercambios no generen una disminución de los costos estos son penalizados para que el intercambio no se vuelva a repetir, de esta forma se logra que el algoritmo converja a una ruta con menores costos cada vez. Cuando la $F A$ tenga valores enteros y el costo sea inferior al costo máximo permitido $\left(C_{M X P}\right)$, observar ecuación (12).

$$
C_{M X P}=n_{C X} *(N-1)
$$

Estaremos frente a la solución del problema, la ruta encontrada y el valor de la función aptitud conformarán el lightpath buscado. Luego se procederá a la actualización de las matrices de costos, lambdas y tiempos, para utilizarse en el siguiente nodo; si los valores de la $F A$ son negativos o decimales se continua iterando; de no encontrarse solución la solicitud es bloqueada.

\section{ESCENARIO DE COMPARACIÓN}

Realizada la simulación se comparó con cuatro trabajos [3] y [8] bajo condiciones de solución integral y [4-5] bajo condiciones de subdivisión del problema.

En la Figura 7 se observa la red NSFNET que fue utilizada en la prueba y posee 16 nodos con 25 enlaces de fibra óptica, los parámetros utilizados fueron similares a los presentados en [5], se realizaron comparaciones de probabilidad de bloqueo y utilización de la red, variando la carga en el intervalo $[0,180]$ con incrementos de 10 Erlangs. El número de conexiones realizadas durante la simulación en [4-5] y el presente trabajo fue de $10^{8}$ solicitudes de conexión.

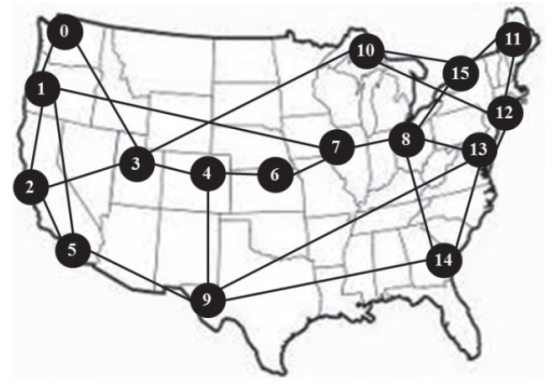

Figura 7. Red NSFENET utilizada. 


\section{RESULTADOS}

Se realizan comparaciones de las simulaciones entre Simulated Annealing [8], Algoritmos Genéticos [3] y los trabajos vistos en [1-2] en las mismas condiciones de simulación con el indicador de probabilidad de bloqueo, que mide la probabilidad de bloquear las solicitudes de servicio bajo una carga determinada, y el indicador utilización de la red que mide la ocupación de la red bajo una carga determinada en Erlangs.

En la Figura 8 se grafica la probabilidad de bloqueo (PB) y podemos observar que TS obtiene una baja PB hasta los 120 Erlangs, superando al resto de algoritmos; esto se debe principalmente a que TS encuentra LP recorriendo una vecindad de soluciones menor al resto de los algoritmos.

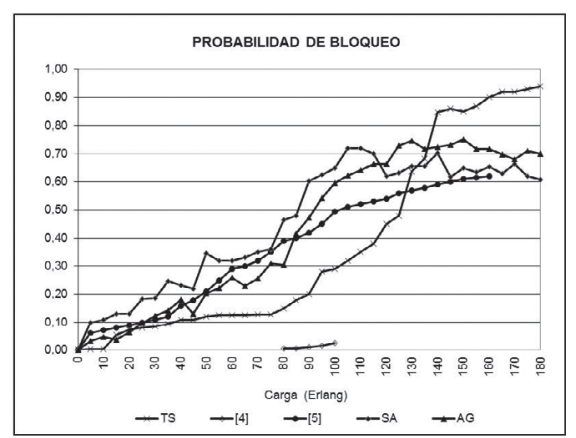

Figura 8. Comparación de la Probabilidad de Bloqueo en la NSFNET.

Sin embargo, por encima de los 120 Erlangs TS no resulta ser el mejor; determinando que para altas cargas de tráfico entrante TS no se desempeña mejor que el resto de algoritmos.

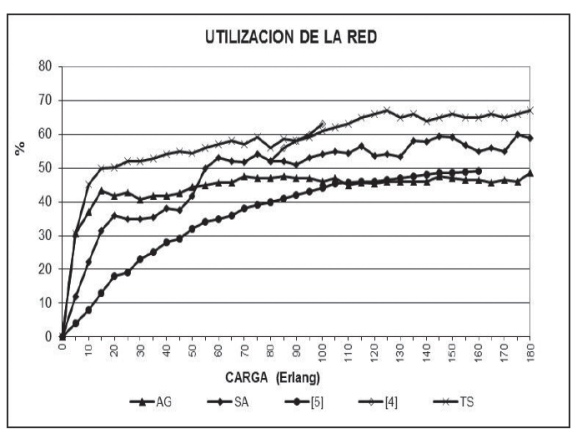

Figura 9. Comparación de la utilización de red en la NSFNET.
No se puede dejar de observar la baja PB del trabajo [4], pero se debe a que se utilizaron 40 longitudes de onda, comparado con las 8 longitudes de onda utilizados por los otros 4 estudios.

En la Figura 9 se muestran las características de los algoritmos respecto de la utilización de la red (UR). El algoritmo TS no es el mejor, y utiliza rápidamente muchos recursos de la red, los otros algoritmos tienen una UR mucho menor, lo que lleva a la reflexión que las rutas obtenidas por el algoritmo TS tienen más saltos que los algoritmos en comparación, lo que tiene como consecuencia una mayor utilización de la red. Del trabajo [4] se desprende que más longitudes de onda no son garantía de eficiencia en la UR, sin embargo ayuda mucho en la disponibilidad y servicio de la red, como se puede observar en la Figura 8.

\section{CONCLUSIONES}

El Grupo de Investigación en Nuevas Tecnologías GINT (USACH-Chile) y el Grupo de Investigación TIGUM (UMNG-Colombia) coinciden que el algoritmo TS tiene un buen comportamiento en lo que respecta al $\mathrm{PB}$, pero un muy bajo comportamiento de la UR, lo que garantiza una buena respuesta al servicio de solicitudes hasta los 120 Erlangs, sin embargo la UR es muy grande, lo que no garantiza un buen servicio de la demanda futura, esto se explica debido a que el algoritmo encuentra rutas más largas. Es necesario seguir investigando en las heurísticas y mejorar las existentes para lograr procesos de PB bajos y UR bajos en condiciones de alta carga, para garantizar los servicios de acceso y transporte dentro de márgenes aceptables, para el internet del futuro. De lo contrario estaremos obligados a seguir aumentando enlaces o desarrollando tecnologías multifibra, que garanticen el servicio.

\section{REFERENCIAS}

[1] N. Charbonneau and V. Vokkarane. "Tabu Search Meta-Heuristic for Static Manycast Routing and Wavelength Assignment over Wavelength-Routed Optical WDM Networks". Communications (ICC), 2010 IEEE International Conference on, pp. 1-5. 2010. ISSN: 1550-3607. DOI: $10.1109 /$ ICC.2010.5502241. 
[2] M. Al-Momin and J. Cosmas. "The Impact of Content Oriented Routing on OpenFlow Burst Switched Optical Networks". Advanced Information Networking and Applications Workshops (WAINA), 2013 27th International Conference on, pp. 965-970. 2013. ISBN: 978-0-7695-4952-1. DOI: 10.1109/ WAINA.2013.139.

[3] A. Rodríguez y F. Saavedra. "Enrutamiento y asignación de longitudes de onda en redes WDM: solución simultánea basada en algoritmos genéticos". IEEE InterconUNSA. Arequipa, Perú. 2009.

[4] X. Chu, B. Li and Z. Zhang. "A Dynamic RWA Algorithm in a Wavelength-Routed All-Optical Network with Wavelength Converters". INFOCOM 2003, TwentySecond Annual Joint Conference of the IEEE Computer and Communications. IEEE Societies. Vol. 3, pp. 1795-1804. 2003. ISSN: 0743-166X. DOI: 10.1109/ INFCOM.2003.1209202.

[5] H. Zang and J. Jue. "Dynamic Lightpath Establishment in Wavelength-Routed WDM Networks". IEEE Communications Magazine. Vol. 39, pp. 100-108. 2001. ISSN: 0163-6804. DOI: $10.1109 / 35.948897$

[6] R. Barpanda, A. Turuk, B. Sahoo and B. Majhi. "Genetic Algorithm techniques to solve Routing and Wavelength Assignment problem in Wavelength Division Multiplexing alloptical networks". Communication Systems and Networks (COMSNETS), 2011 Third International Conference on, pp. 1-8, 2011. ISBN: 978-1-4244-8951-0. DOI: 10.1109/ COMSNETS.2011.5716507.

[7] A. Rodríguez, F. Saavedra y L. Ramírez. "Solución Simultánea del Enrutamiento y Asignación de Longitud de Onda en redes WDM con Algoritmos Genéticos”. IEEE COLCOM. Bogotá, Colombia. 2008.

[8] A. Rodríguez, F. Saavedra and L. Ramírez. "Simulated Annealing una Propuesta de Solución al Problema RWA en Redes Fotónicas". IEEE InterconUNI. Lima, Perú. 2011.

[9] H. Guenduez and H. Kadir. "A WellArranged Simulated Annealing Approach for the Location-Routing Problem with Time Windows". System Sciences (HICSS). 2013 46th Hawaii International Conference on, pp. 1144-1153. 2013. ISSN: 1530-1605. DOI: 10.1109/HICSS.2013.69.

[10] J. Kuri, N. Puech, M. Gagnaire, E. Dotaro and R. Douville. "Routing and wavelength assignement of scheduled lightpath demands". IEEE Journal On Selected Areas in Communications. Vol. 21, pp. 1231-1240. 2003. ISSN: 0733-8716. DOI: 10.1109/ JSAC.2003.816622.

[11] C. Yi-Jen, W. Tsu-Hsiu and W. Jui-Pin. "High-speed semiconductor all-optical conversiondevice based on field-driven quantum well". Advanced Infocomm Technology (ICAIT), 2013 6th International Conference on, pp. 147-148. 2013. ISBN: 978-1-4799-0464-8. DOI: 10.1109/ ICAIT.2013.6621538.

[12] K. Bhaskaran, J. Triay and V. Vokkarane. "Dynamic Anycast Routing and Wavelength Assignment in WDM Networks Using Ant Colony Optimization (ACO)". Communications (ICC), 2011 IEEE International Conference on, pp. 1-6. 2011. ISSN: 1550-3607. DOI: 10.1109/ icc.2011.5962826.

[13] T. Ramesh, N. Amrutha, A. Madhu, K. Saumya and P. Vaya. "A Proactive and Self-Regulated Ant-Based RWAProtocol for All-Optical WDM Networks". Process Automation, Control and Computing (PACC), 2011 International Conference on, pp. 1-5. 2011. ISBN: 978-1-61284-765-8. DOI: 10.1109/PACC.2011.5978978.

[14] S. Ngo, J. Xiaohong and S. Horiguchi. "Adaptive routing and wavelength assignment usingant-based algorithm". Networks, 2004. (ICON 2004). Proceedings. 12th IEEE International Conference on. Vol. 2, pp. 482-486. ISSN: 1531-2216. DOI: 10.1109/ ICON.2004.1409214.

[15] N. Skorin-Kapov. "Heuristic algorithms for the routing and wavelength assignment of scheduled lightpath demands in optical networks". Selected Areas in Communications, IEEE Journal on. Vol. 24 , pp. 2-15. 2006. ISSN : 0733-8716. DOI: 10.1109/ JSAC.2006.1677251.

[16] D. O'Brien, B. Chatelain, F. Gagnon and C. Tremblay. "A Dual Metaheuristic Solution to the Min-RWA Problem". Optical Fiber communication/National Fiber 
Optic Engineers Conference. 2008. OFC/ NFOEC 2008. Conference on, pp. 1-3. 2008. ISBN: 978-1-55752-856-8. DOI: 10.1109/ OFC.2008.4528114.

[17] C. Dzongang, P. Galinier and S. Pierre. "A tabu search heuristic for the routing and wavelength assignment problem in optical networks". Communications Letters, IEEE. Vol. 9, pp. 426-428. 2005. ISSN: 1089-7798. DOI: 10.1109/LCOMM.2005.05011.

[18] D. Khyda, S. Chamberland and S. Pierre. "Improvement of routing and wavelength assignment in WDM networks using tabu search". Electrical and Computer Engineering, 2003. IEEE CCECE 2003. Canadian Conference on. Vol. 2, pp. 765768. 2003. ISSN: 0840-7789. DOI: $10.1109 /$ CCECE.2003.1226007.
[19] W. Ying, C. Tee and L. Meng. "A Tabu search algorithm for static routing and wavelength assignment problem". Communications Letters, IEEE. Vol. 9, pp. 841-843. 2005. ISSN: 1089-7798. DOI: $10.1109 /$ LCOMM.2005.1506721.

[20] F. Glover. "Tabu Search, Part I". ORSA Journal on Computing. Vol. 1, Issue 3, pp. 190-206. 1989.

[21] F. Glover. "Tabu Search, Part II". ORSA Journal on Computing. Vol. 2, Issue 1, pp. 4-32. 1990.

[22] A. Rodríguez y F. Saavedra. "Optimización del Algoritmo Genético para la Solución Integral de Enrutamiento en Redes Fotónicas". Información Tecnológica. Vol. $21 \mathrm{~N}^{\mathrm{o}} 3$. 2010. ISSN: 0718-0764. DOI: $10.4067 /$ S0718-07642010000300014. 